\title{
B. URLI
}

\section{BEAUDRY}

\section{Une approche multicritère d'allocation des ressources financières dans le domaine de la santé}

Revue française d'automatique, d'informatique et de recherche opérationnelle. Recherche opérationnelle, tome 29, no 4 (1995), p. 373-389.

<http://www.numdam.org/item?id=RO_1995_29_4_373_0>

(C) AFCET, 1995, tous droits réservés.

L'accès aux archives de la revue « Revue française d'automatique, d'informatique et de recherche opérationnelle. Recherche opérationnelle » implique l'accord avec les conditions générales d'utilisation (http://www.numdam.org/ legal.php). Toute utilisation commerciale ou impression systématique est constitutive d'une infraction pénale. Toute copie ou impression de ce fichier doit contenir la présente mention de copyright.

\section{Numdam}

Article numérisé dans le cadre du programme

Numérisation de documents anciens mathématiques

http://www.numdam.org/ 
Recherche opérationnelle/Operations Research

(vol. $29, \mathrm{n}^{\circ} 4,1995$, p. 373 à 389)

\title{
UNE APPROCHE MULTICRITÈRE D'ALLOCATION DES RESSOURCES FINANCIĖRES DANS LE DOMAINE DE LA SANTÉ (*)
}

\author{
par B. URLi $\left({ }^{1}\right)$ et D. Beaudry $\left({ }^{2}\right)$ \\ Communiqué par Jean-Pierre BRANS
}

\begin{abstract}
Résumé. - Depuis plusieurs années, le Ministère de la Santé et des Services Sociaux (M.S.S.S.) alloue annuellement aux différents centres régionaux (C.R.S.S.S.) des budgets de développement à des fins très précises et sous la forme d'enveloppes protégées dites par programme. La répartition équitable de ces sommes à l'intérieur d'une région donnée devient fort complexe et se doit de tenir compte des populations desservies et de l'état de santé de ces populations. Pour répondre à cette problématique, nous avons développé une méthode d'allocation qui s'appuie sur le concept de "santé globale», concept fondamentalement multidimensionnel, pour opérationnaliser cette notion d'équité dans l'allocation des budgets. Dans cette perspective, l'utilisation d'une approche constructive d'aide à la décision multicritère était bien adaptée à notre problème. Cet aspect "constructif» de l'approche retenue était très important et ceci particulièrement en regard du contexte très politique de cette décision d'allocation. Dans cette communication, nous présentons de quelle manière une modification des méthodes d'AHP et de PROMETHEE ont permis d'en arriver $\grave{a}$ une proposition d'allocation et nous formulons quelques remarques quant à l'acceptation, par les gestionnaires. impliqués, de cette approche constructive d'aide à la décision.
\end{abstract}

Mots clés : Méthodologie multicritère ; allocation des ressources ; approche constructive.

Abstract. - For some years, the Quebec Health and Welfare Ministry allocates annual funds for specific development programs in different administrative regions of Quebec, Canada. Equitable allocation of these budgets to different social establishment of a given administrative region becomes complex. It depends on the populations to serve and on the health of these populations. In response to that problematic, we have developped a method which relies on the notion of "global health" to maintain equitable allocation. It soon appeared that several points of view had to be considered and that the use of a multicriteria decision aid method in a constructive approach was well-adapted to this problem. This "constructive" aspect of our approach was very important here because of the political context of that problematic. In this paper, we related how modifications of the AHP and PROMETHEE methods were used. We describe how the criteria were defined and how the weights were obtained through the application of that methodology to the program "personnes âgées en perte d'autonomie". Finally, we summarize how real-world decision makers accepted the method.

Keywords: Multicriteria methodology; ressource allocation; constructive approach.

(*) Reçu en juin 1992.

(1) Université du Québec à Rimouski.

$\left({ }^{2}\right)$ Conseil Régional de la Santé et des Services Sociaux 01-11.

Recherche opérationnelle/Operations Research, 0399-0559/95/04/\$4.00

(C) AFCET-Gauthier-Villars 


\section{CADRE GÉNÉRAL}

Les besoins en santé de même que les problèmes existant dans le domaine de la santé, diffèrent d'une région à l'autre du Québec. Comment d'ailleurs pourrait-il en être autrement puisque l'on sait que ces populations sont loin d'être homogènes sur le plan démographique (distribution différente des populations selon l'âge et le sexe, taux de mortalité plus ou moins élevé, etc.), sur le plan économique (taux de chômage inégal, scolarisation différenciée, etc.) et sur le plan de la morbidité (prévalence et incidence du cancer, de maladies respiratoires, des maladies cardio-vasculaires, etc.). Ces simples considérations posent déjà tout le problème que connait régulièrement le ministère de la Santé et des Services sociaux (M.S.S.S.) dans 1'allocation des budgets aux diverses régions socio-sanitaires de la province. Ce problème se pose encore avec plus d'acuité aujourd'hui compte-tenu du contexte de régionalisation des budgets.

$\mathrm{Au}$ milieu des années 80, conscient qu'il devait prendre en compte les particularités régionales, le M.S.S.S. [8] a développé un modèle d'allocation des ressources financières inter-régionales. L'objectif visé par l'utilisation de ce modèle était évidemment de tendre vers une allocation équitable des ressources financières entre les régions. Utilisé depuis lors pour procéder aux répartitions budgétaires intra-régionales dans le cadre de certains programmes de santé, ce modèle mieux connu sous le vocable de « Grille Lamarche », traduit un souci légitime d'adéquation des ressources financières aux besoins des populations.

Si cette façon d'aborder le problème est en soi meilleure que l'absence de méthodologie ou encore, de l'utilisation du per capita, il demeure que bien des critiques ont été faites sur l'utilisation de cette méthode, notamment par N. Berthiaume [2]. Parmi l'ensemble de celles-ci, quelques-unes méritent d'être examinées :

1) La santé d'une population et les besoins qui lui sont inhérents ne peuvent être évalués sur la seule base des services « consommés » comme le fait la « Grille Lamarche ». En effet, les régions périphériques ne disposant pas de tous les services disponibles dans les grands centres, ce raisonnement conduit à penser que les services sont absents parce que la population n'a aucun besoin à ce chapitre. Poussé à l'extrême, ce raisonnement peut empêcher tout développement de services dans les régions périphériques.

2) Cette méthode ne tient nullement compte des variations de coûts qui existent pour un même service entre les diverses régions. A titre d'exemple, 
citons le cas d'une infirmière visiteuse qui aura des distances beaucoup plus grandes pour rejoindre les bénéficiaires dans une région périphérique, qu'il n'en serait si elle travaillait à Montréal ou Québec. Pour une région comme l'Abitibi-Témiscamingue, ces distances se répercutent sur le coût du service.

3) Une définition restreinte de la santé, puisque seule la consommation de services et de soins est considérée pour évaluer l'état de santé de la population. Or, ce ne sont pas tous les problèmes de santé qui font l'objet d'une demande de soins. Qui plus est, ce ne sont pas toutes les demandes de soins qui font l'objet d'une demande de services de santé. Par conséquent, tout modèle qui ne s'appuierait que sur les services réellement utilisés ne pourrait être que réductionniste dans sa tentative d'obtenir la meilleure adéquation possible entre les besoins en santé des populations et les ressources allouées pour satisfaire ces besoins. En plus d'une dimension économique, la santé recouvre des dimensions humaine et sociale, que la grille Lamarche ne prend pas en compte.

En effet, il est désormais entendu par l'ensemble du milieu de la santé que la notion même de santé ne recouvre plus que le seul état physique d'un individu ou d'une population. Ainsi, à cette traditionnelle perception biologique de ce qu'était la santé, se sont greffées d'autres dimensions qui sont sociale, psychologique et environnementale. Le rapport Lalonde, dans une brochure parue en 1975 et portant sur la santé des canadiens, reconnaissait déjà la santé comme un état de bien-être physique qui englobait aussi les dimensions sociales et mentales. Les années passèrent et au fur et à mesure que la réflexion sur la question avançait, on vit apparaître une définition de la santé, S. Dillard [7], qui était celle d'un processus d'adaptation des individus à leur milieu.

Cette définition qui est acceptée actuellement par l'ensemble du milieu de la santé, suppose que cette dernière est la résultante de l'interaction de l'individu avec son environnement. On ne considère donc plus la dimension biologique comme la seule composante de la santé mais plutôt comme un élément parmi d'autres. Partant de là, divers déterminants de l'état de santé ont été identifiés. Ces déterminants sont, à des degrés divers et sous différentes formes, capables d'influencer la qualité de la santé de l'individu et par conséquent, des populations elles-mêmes. Parmi ceux-ci, on retrouve les suivants :

- l'environnement économique et social dans lequel coexistent le ou les individus (chômage, scolarisation, etc.) ; 
- les caractéristiques du système de soins et services du milieu (structure, coût, etc.) ;

- les caractéristiques démographiques de l'environnement (âge des populations, le pourcentage de plus de 65 ans dans la population) ;

- les habitudes de vie des populations (exercice physique, proportion de fumeurs, etc.).

Ainsi, la notion de santé des populations apparaît dans un cadre multidimensionnel qui tient à la fois compte de l'environnement économique, démographique, social et bien sûr, biologique. Dans une telle perspective, il apparaît évident que la distribution équitable d'une enveloppe budgétaire à des fins de maintien ou d'amélioration de l'état de santé ne peut exclure l'une ou l'autre de ces dimensions.

A l'instar du M.S.S.S. qui alloue certains budgets par programme aux régions, les conseils régionaux de la santé et des services sociaux (C.R.S.S.S.) doivent à leur tour procéder à une distribution équitable de ces enveloppes aux sous-régions dont ils ont la responsabilité. Ainsi, la difficulté que peut rencontrer le M.S.S.S. dans la recherche d'une allocation équitable des budgets aux régions est vécue également par les C.R.S.S.S. qui, animé du même souci d'équité, doivent répartir entre les divers établissements les budgets reçus.

En réponse à cette problématique, nous avons développé une méthodologie multicritère d'allocation des ressources financières régionales dont le principe directeur est le principe d'équité. Cette méthodologie recourt à une évaluation multidimensionnelle de cette notion d'équité par le truchement de différents indicateurs, chacun d'eux décrivant et estimant une dimension particulière de ce concept d'équité.

Par ailleurs, en plus de cette multidimensionnalité du problème, il est important de noter que ce processus d'allocation baigne dans un contexte fortement politique car l'implantation d'une nouvelle méthodologie signifie à la fois une révision en profondeur des habitudes d'attributions des budgets de développements aux établissements dispensant des services de santé et services sociaux à la population et, des relations que ceux-ci entretenaient avec le pourvoyeur de ces fonds, c'est-à-dire le Conseil régional de la santé et des services sociaux (C.R.S.S.S.). Conséquemment, l'acceptation des nouveaux paramètres d'attribution demeurait la partie du projet comportant la part la plus importante d'impondérables. En effet, bien que les gestionnaires des divers établissements jugeaient important de se doter d'une grille d'allocation «objective », ceux-ci ne voyaient pas d'un œil favorable 
l'avènement d'une « technique mathématique » qui, disaient-ils, viendraient les priver de toute capacité d'influencer le système d'attribution des budgets de développement. En d'autres mots, bien que tous s'entendaient sur la nécessité de se doter d'une nouvelle méthodologie, chacun souhaitait que celle-ci tienne compte de la réalité qui lui était propre. Toute modélisation qui ne pourrait traduire leur perception de la réalité ne pourrait s'avérer qu'un échec.

C'est ainsi, que l'utilisation d'une approche constructive d'aide à la décision fut mise de l'avant pour essayer de canaliser et de faire converger les perceptions donc, les intérêts des différents acteurs, rendant ainsi le projet réalisable sur le plan politique. En effet, le gestionnaire appelé à gérer un projet dont la dimension politique peut à tout moment compromettre, voire stopper le déroulement des travaux doit suivre l'évolution de l'univers dans lequel baigne le projet. A l'instar de la dialectique sujet-objet décrite par Piaget, le gestionnaire doit constamment valider avec les différents groupes d'acteurs du projet si ce dernier réfléchit dans sa construction les préoccupations et réalités des différents groupes d'intérêts.

\section{LA MÉTHODOLOGIE MULTICRITÈRE D'ALLOCATION DES RESSOURCES}

Cette méthodologie vise à une allocation équitable des ressources entre les territoires de M.R.C. (Municipalité Régionale de Comté) en s'appuyant sur l'état de santé des populations. Cette notion de santé des populations est fondamentalement multidimensionnelle et il est aujourd'hui accepté par l'ensemble du milieu de la santé qu'il existe différents déterminants capables d'influencer la qualité de la santé de l'individu et par conséquent des populations. Dans une telle perspective, la méthodologie proposée prend en compte cet aspect multidimensionnel de l'état de santé et s'articule autour des points suivants :

Construction du modèle d'allocation

Obtention des poids

Agrégation des évaluations

Recommandations pour l'allocation 


\subsection{La construction du modèle}

Le modèle proposé retient, après validation auprès d'experts, un certain nombre de dimensions pour son appréciation. Chacune de ces dimensions est elle-même décrite et estimée par des indicateurs qui ont été retenus par des experts. Le modèle utilisé est donc un construit dont la validité repose sur un arbitrage, réalisé par les comités d'experts, entre validité interne et externe. Le modèle général prend la forme (fig. 1) qui suit :

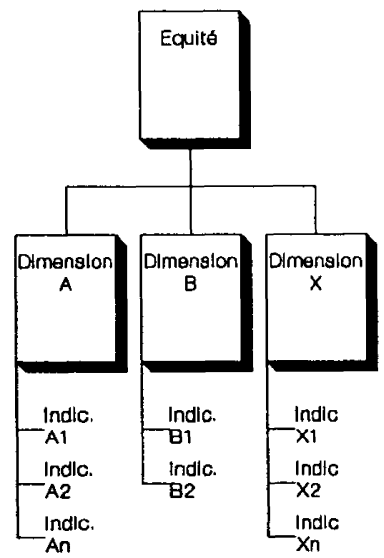

Figure 1. - Le modèle général d'allocation, un construit.

\section{Le découpage en unités territoriales}

Il était nécessaire de déterminer, dans un premier temps, l'unité à laquelle allait s'adresser l'allocation financière. L'unité territoriale retenue fut la M.R.C. Plusieurs raisons militaient en faveur de ce choix : décentraliser une partie de la décision au niveau sous-régional en favorisant la concertation des établissements, pouvoir disposer d'indicateurs adéquats (fiables, valides et alimentés par des sources d'information reconnues). A ce titre, la méthodologie proposée s'adresserait d'autant mieux à une allocation interrégionale car l'on disposerait alors d'une banque d'indicateurs encore plus riche.

\section{La structuration hiérarchique du problème}

Eu égard à la structuration hiérarchique (fig. 1) de la problématique d'allocation de ressources, plusieurs comités d'experts furent formés. Ces 
comités étaient « étanches », c'est-à-dire qu'un individu ne pouvait siéger que sur un seul comité. Selon la nature du comité, celui-ci pouvait être appelé à choisir et pondérer les dimensions (dimension démographique, socio-économique, etc.) ou choisir et pondérer les indicateurs retenus (taux d'innocupation, taille des populations, etc.) pour décrire et estimer ces grandes dimensions. De fait, on distingue trois types de comités :

- le comité «des directeurs » dont le rôle consiste à sélectionner, à valider les dimensions de l'équité et à leur accorder une importance relative. Il est composé des directeurs généraux des établissements et des organismes communautaires concernés par le programme pour lequel est effectué l'allocation ;

- le comité " programme » dont le rôle consiste à sélectionner, parmi une liste présentée par le groupe de travail, à valider et à pondérer les indicateurs relatifs à la dimension " programme », en l'occurrence celui des " personnes âgées en perte d'autonomie ». Ce comité comprend des personnes reconnues, par les membres du comité des directeurs, comme expertes relativement à la problématique plus particulière des personnes âgées en perte d'autonomie ;

- les comités « hors-programme » qui jouent un rôle semblable à celui du comité «programme » mais pour ce qui a trait au autres dimensions considérées comme pertinentes à la problématique. Ces comités sont composés de personnes qui s'intéressent à la problématique globale de santé des personnes âgées. Ces personnes ont toutes été proposées et acceptées à l'unanimité comme expertes par les membres du comité des directeurs.

Le modèle retenu pour l'allocation des ressources relatives au programme des personnes âgées en perte d'autonomie est situé à l'annexe 1 .

\subsection{L'obtention des poids relatifs aux dimensions et aux indicateurs d'Équité}

Pour colliger les données des experts des différents comités susmentionnés, nous avons utilisé l'AHP de T. Saaty [9]. Cette technique nous permettrait notamment d'utiliser comme critère de tamisage des experts, les seuils de cohérence générés à partir des matrices de comparaisons binaires. $\mathrm{Si}$ ces niveaux n'évaluent que la cohérence en terme de transitivité des jugements, n'oublions pas que leur expertise a été validée par la décision unanime des membres du comité des directeurs. Cependant, comme ces informations sont colligées auprès de chaque expert, il est alors nécessaire d'agréger les jugements de ces experts en une matrice synthèse. Pour ce 
faire, nous avons retenu la démarche suivante :

Présentation des contenus sémantiques des dimensions ou indicateurs

Remplissage des matrices de comparaisons binaires de manière individuelle

Tamisage des matrices à partir d'un seuil minimal de cohérence

Analyse de cette matrice agrégée pour évaluer le consensus

Construction d'une matrice agrégée à partir d'une moyenne pondérée par les niveaux de cohérence

\section{Calcul des poids}

Dans un premier temps, les experts recevaient un tableau récapitulatif des discussions effectuées lors de l'étape de structuration des dimensions ou des indicateurs. On y retrouvait alors des informations sur les dimensions ou les indicateurs validés par l'ensemble des experts du comité en question, à savoir une description littérale de la dimension ou de l'indicateur, la source d'information concernant la dimension ou l'indicateur, sa méthode de calcul ainsi que son sens de variation (maximum ou minimum) lorsqu'il s'agissait d'un indicateur. Par la suite, et de manière individuelle, les experts remplissaient une matrice de comparaisons binaires des dimensions ou des indicateurs validés. Celles-ci étaient alors traitées au moyen d'un module de calcul des poids intégré au sein du logiciel AMARRE-CALC qui supporte la méthode d'allocation que nous présentons dans cet article. Dans cette procédure de calcul des poids, un tamisage des matrices est effectué à partir d'un seuil minimal de cohérence. Une analyse de la distribution des valeurs des matrices retenues était réalisée pour évaluer le consensus entre les experts. La dispersion des valeurs des experts a été retenu comme mesure de ce consensus. Enfin, les matrices retenues étaient agrégées en une matrice synthèse à partir d'une moyenne pondérée par les niveaux de cohérence propres à chacune des matrices retenues et le calcul des poids à partir de cette matrice synthèse était réalisé. 
Dans cette procédure, l'étanchéité des comités était importante. En effet, pour limiter un jeu trop évident dans la pondération, les poids des indicateurs n'étaient pas remis aux membres qui pondéraient les dimensions. Dans le cadre de notre application au programme des personnes âgées en perte d'autonomie, cet exercice de validation et de pondération des dimensions et des indicateurs nous a fourni les résultats de l'annexe 2 . On notera que la valeur maximale du ratio de cohérence retenu ici était de $15 \%$, ceci parce que cette allocation constituait une première utilisation de ce type de méthode. Dans l'avenir, ce niveau devra être inférieur ou égal à $10 \%$.

\subsection{L'agrégation des évaluations}

Ce construit, qui est une représentation validée par différents comités d'experts de la problématique d'allocation équitable des ressources intrarégionales, permet alors d'évaluer la «performance » de chacune des unités territoriales en regard de chacun de ces indicateurs. On dispose alors d'une matrice d'évaluation avec les valeurs $V_{j}(i)$ (Évaluation de l'unité territoriale $i$ sur l'indicateur $j$ ), et des poids relatifs $\left(w_{j}\right)$ des différents indicateurs $j$.

Maintenant, la performance d'une unité territoriale donnée sera généralement forte sur un indicateur particulier et plus faible sur un autre. Pour évaluer la performance globale d'une unité territoriale, il est alors nécessaire d'agréger les évaluations $V_{j}(i)$. Dans les publications portant sur l'analyse multicritère, on retrouve beaucoup de méthodes d'agrégation. On peut distinguer celles qui relèvent d'une agrégation totale (par l'utilisation d'une fonction multiattribut), d'une aggrégation partielle (en recourant aux relations de surclassement) ou d'une agrégation locale et interactive (avec la programmation multiobjectif). Dans le cadre de notre application, la nature des échelles de mesure propre à chaque indicateur nous a amené à opter pour l'utilisation des relations de surclassement. Parmi toutes les méthodes basées sur les relations de surclassement (Schärlig [10], Vincke [11]), nous avons choisi la méthode PROMETHEE (Brans, Vincke et Mareschal [4]), méthode que nous avons modifiée pour satisfaire les caractéristiques de notre problème. La simplicité et la facilité de présentation des concepts sous-jacents à la méthode sont à la base de notre choix.

La méthode PROMETHEE est basée sur la construction d'une relation de surclassement valué dans l'ensemble des actions A (les unités territoriales) et elle permet de générer des préordres partiels de telle façon que certaines des unités territoriales peuvent être incomparables. Dans PROMETHEE II, on exploite aussi cette relation de surclassement pour générer un préordre 
total unique des unités territoriales. Pour tout couple $(a, b) \in A \times A$ on définit une fonction $P_{j}(a, b)$ qui traduit « l'intensité » de préférence de $a$ sur $b$ pour l'indicateur $j$. Cette fonction est du type :

$$
P_{j}(a, b)=\left\{\begin{array}{ccc}
0 & \text { si } & V_{j}(a) \leq V_{j}(b) \\
F_{j}(a, b) & \text { si } & V_{j}(a)>V_{j}(b)
\end{array}\right.
$$

où $F_{j}(a, b)$ est une fonction croissante de $\left[V_{j}(a)-V_{j}(b)\right]$. Six formes de courbes $F_{j}(a, b)$ ont été proposées (Brans, Vincke et Mareschal [4]) bien que d'autres pourraient éventuellement être introduites.

On génère alors un indice de préférence multicritère

$$
\Pi(a, b)=\sum_{j} w_{j} \cdot P_{j}(a, b), \quad x_{j} \geq 0 .
$$

Enfin, pour ranger les unités territoriales, on définit les flux de préférence normés qui suivent :

$$
\begin{aligned}
\Phi^{+}(a) & =(1 / n-1) \sum_{b \in A} \Pi(a, b) & & \text { flux sortant normé } \\
\Phi^{-}(a) & =(1 / n-1) \sum_{b \in A} \Pi(b, a) & & \text { flux entrant normé } \\
\Phi(a) & =\Phi^{+}(a)-\Phi^{-}(a) & & \text { flux net normé }
\end{aligned}
$$

et on peut produire le préordre total : $a$ est Préféré à $b$ si $\Phi(a)>\Phi(b)$, et $a$ est Indifférent à $b$ si $\Phi(a)=\Phi(b)$. Dans le cadre de notre application, on a été amené à proposer deux modifications à cette méthode d'agrégation.

\section{Utilisation d'écart en relatif}

Dans la méthode PROMETHEE, les courbes d'intensité de préférence $F_{j}(a, b)$ sont des fonctions croissantes des écarts dans les évaluations, indépendamment de l'amplitude des évaluations. Cependant, la signification d'une différence de 1000 habitants n'est pas la même selon que l'on considère des populations en milliers ou en dizaines de milliers d'habitants. Conséquemment, nous avons déterminé les critères généralisés comme des fonctions croissantes non pas de l'écart absolu $\left[V_{j}(a)-V_{j}(b)\right]$ mais plutôt de l'écart relatif $\left[\left(V_{j}(a)-V_{j}(b)\right) /\left(\left(V_{j}(a)+V_{j}(b)\right) / 2\right)\right]$.

Utilisation des solutions idéale et anti-idéale

Nous avons défini les seuils de préférence $p_{j}$ comme l'écart relatif maximum entre les évaluations sous l'indicateur $j$, soit

$$
p_{j}=\left(V_{j}\left(x^{*}\right)-V_{j}\left(x_{*}\right)\right) /\left(\left(V_{j}\left(x^{*}\right)-V_{j}\left(x_{*}\right)\right) / 2\right)
$$


où $V_{j}\left(x^{*}\right)$ correspond à la meilleure évaluation obtenue pour l'ensemble des unités territoriales de $A$ relativement à l'indicateur $j$. De même, $V_{j}\left(x_{*}\right)$ peut être considérée comme la valeur anti-idéale, c'est-à-dire la moins préférée en regard de toutes les autres valeurs de l'indicateur $j$. Conséquemment, $P_{j}(a, b)$ examine la position des deux unités territoriales $a$ et $b$ non seulement l'une envers l'autre, mais aussi en regard de toutes les unités territoriales de $A$. Sa valeur est toujours comprise dans l'intervale $[0,1]$ elle est égale à 1 si et seulement si $V_{j}(a)=V_{j}\left(x^{*}\right)$ et $V_{j}(b)=V_{j}\left(x_{*}\right)$. $\Pi(a, b)$, quant à lui, traduit l'indice de préférence de l'unité $a$ sur l'unité $b$ et le flux net normé d'une unité territoriale $a(\Phi(a))$ représente alors une mesure appropriée de la supériorité de $a$ sur toute autre unité territoriale $b$ de $A$. Utilisant une procédure semblable, à tout le moins pour la définition de l'indice de préférence stricte SP $(a, b)$, Diakoulaki et Koumoutsos [6] estiment que l'on peut exploiter les flux nets non seulement pour le rangement des actions (unités territoriales) mais aussi pour la construction d'une échelle cardinale. Notons par ailleurs, en ce qui a trait à l'utilisation de seuils d'indifférence $q_{j}$, comme dans le cas de fonctions de type linéaire avec seuil, ce seuil était fixé comme un pourcentage du seuil de préférence $p_{j}$. L'utilisation d'une telle procédure de fixation des seuils nous amène alors à associer des valeurs numériques significatives aux flux nets normés des différentes unités territoriales. La signification des valeurs des flux nets n'est bien évidemment pas absolue mais relative car elle est directement liée à l'ensemble particulier d'unités territoriales $\{A\}$.

\subsection{Recommandations pour l'allocation}

Notre démarche de modélisation se situe dans le cadre d'une méthodologie d'aide à la décision visant à éclairer le Comité de gestion dans son rôle décisionnel d'allocation des ressources. Pour ce faire, deux attitudes peuvent caractériser ce cheminement vers l'équité : une attitude "brutale » où l'on vise à corriger le plus rapidement les écarts d'équité entre les différentes unités territoriales ou une autre, plus «harmonieuse », qui fixe un pas (seuil) dans cette course vers l'équité. Nous avons proposé de déterminer le pourcentage alloué à chaque unité territoriale par l'équation suivante :

$$
\% \text { alloué }(i)=\left(\Phi(i)-\Phi_{\min }\right) / \sum_{j}\left(\Phi(j)-\Phi_{\min }\right)
$$

Ceci, pour que l'unité territoriale ayant le flux net le plus faible $\left(\Phi_{\min }\right)$, celle qui a le moins besoin de ressources financières compte tenu du principe d'équité retenu, ne reçoive rien. Cependant, cette démarche peut 
paraître un peu brutale d'un point de vue social et il est possible d'en suivre une autre, plus harmonieuse, en fixant un seuil (une allocation minimale) dans cette course vers l'équité. C'est pourquoi l'utilisation de seuils minimums sur les pourcentages alloués et/ou sur les montants à allouer on été pris en considération dans les recommandations pour aider les gestionnaires du réseau de la santé et des services sociaux dans leur décision d'allocation des ressources financières. Ces possibilités ont été introduites dans un module de gestion au sein du logiciel AMARRE-CALC qui supporte l'Approche Multicritère d'Allocation des Ressources REgionales que nous avons présentée dans de document.

On peut retrouver en annexe 3 les résultats obtenus dans le cadre de notre application au programme des personnes âgées en perte d'autonomie. Notons que les fonctions retenues étaient toutes de type linéaire pour ce qui a trait aux indicateurs et qu'une allocation minimale de 55,000\$ a été retenue par les gestionnaires du C.R.S.S.S. Cette allocation minimale a été considérée de manière à ce que cette course vers l'équité prenne en compte une dynamique historique en facilitant une transition avec les allocations antérieures.

\section{CONCLUSION}

La démarche méthodologique proposée est avant tout constructive (D. Bouyssou [3]) et il en est de la validité et de l'acceptation du modèle que cette construction soit effectuée avec soin. A ce titre, la démarche de consultation auprès des comités d'experts est essentielle et il faut faire en sorte que ceux-ci disposent de l'information et du temps nécessaires pour valider et pondérer les dimensions et indicateurs relatifs au modèle. Cette démarche de consultation et de validation continuelle facilite l'appropriation de la méthode d'allocation par les membres concernés du réseau de la santé et des services sociaux. Notons qu'il est essentiel de mettre à jour et de tenter d'améliorer sans cesse les indicateurs utilisés. A ce titre, la mise sur pied de groupes de travail sur la création et/ou la validation de bons indicateurs au niveau des M.R.C. serait souhaitable. Remarquons par ailleurs que cette difficulté à avoir des indicateurs validés scientifiquement semble induire chez les experts une tendance à sur-évaluer les dimensions et indicateurs qui ne rencontrent pas cette difficulté.

L'utilisation que nous avons faite de la méthode de l'AHP a permis de bâtir une représentation validée de la problématique d'allocation des ressources. Cette démarche, intégrant un cloisonnement des comités d'expert pour limiter le jeu politique et différents niveaux de validation des dimensions 
comme des indicateurs et de leurs poids relatifs, a grandement facilité la convergence des intérêts des différents acteurs rendant ainsi réalisable ce projet sur le plan politique. Quant à la méthode d'agrégation elle-même, nous avons retenu la méthode PROMETHEE. Celle-ci se caractérise par la simplicité de ses concepts, de sa procédure de calculs et de sa capacité à modéliser les problèmes par le recours aux critères généralisés. Les modifications que nous avons apportées à cette méthode concernent la prise en compte de l'amplitude des évaluations dans la définition des fonctions critères généralisés et l'utilisation des solutions idéales et anti-idéale dans la détermination de la position relative des actions comparées en regard de l'ensemble des actions examinées. Ces modifications ont permis de réaliser une recommandation d'allocation qui satisfait les caractéristiques de notre application sans alourdir la méthode.

\section{BIBLIOGRAPHIE}

1. D. Beaudry, A. Gbodossou et B. Urli, Modèle d'allocation des ressources, Conseil régional de la santé et des Services sociaux de l'Abitibi-Témiscamingue, 1990, Rouyn-Noranda.

2. N. Berthiaume, Critique préliminaire du modèle d'allocation des ressources consenties par le ministère de la Santé et des Services sociaux, Conseil Régional de la santé et des services sociaux de l'Abitibi- Témiscamingue, mars 1988, Rouyn-Noranda.

3. D. Bouyssou, Approches descriptives et constructives d'aide à la décision. Fondements et décision, Thèse de doctorat, U.E.R. Science des organisations, 1984, Université de Paris-Dauphine.

4. J. P. Brans, P. Vincke et B. Mareschal, How to select and how to rank project: the promethee method, 1986, EJOR 24, p. 228-238.

5. R. Chonmere, R. Malenfant, Y. Sauvageau, E. Cadieu, Liste des indicateurs pour le réseau des DSC, Comité provincial des CH-DSC, DSC de l'hôpital général de Montréal, 1990, Montréal.

6. D. Diakoulaki et N. Koumoutsos, Cardinal Ranking of Alternative Actions : Extension of the PROMETHEE Method, EJOR 53, 1991, p. 337-347.

7. S. Dillard, Une analyse multivariée de l'environnement socio-économique de la santé, Conseil des Affaires sociales et de la famille, Québec, 1985.

8. Direction générale de la planification et de l'évaluation, Modifications suggérées aux critères d'allocation des ressources consenties par le ministère de la Santé et des Services sociaux au réseau des Affaires sociales, M.S.S.S., Québec, juin 1987.

9. T. L. SAATY, The Analytic Hierarchy Process, McGraw-Hill, 1980, New York.

10. A. Schärlig, Décider sur plusieurs critères, Presses Polytechniques Romandes, 1985.

11. Ph. VINCKE, L'aide multicritère à la décision, Ellipses, 1989, Paris. 
ANNEXE 1

Modèle d'allocation personnes âgées en perte d'autonomie dimensions et indicateurs validés

\begin{tabular}{|c|c|c|c|c|}
\hline \multirow{2}{*}{ Dimensions } & \multirow{2}{*}{ Indicateurs } & \multirow{2}{*}{ Descriptions } & \multicolumn{2}{|c|}{ Sens } \\
\hline & & & Maximal & Minimal \\
\hline \multirow{3}{*}{ Démographique . } & Taux variation & $\begin{array}{l}\text { Taux de variation } \\
\text { des populations. }\end{array}$ & $\mathrm{X}$ & \\
\hline & Taille Pop. & Taille des populations. & & $\mathrm{X}$ \\
\hline & Rapport $<15 />65$ & $\begin{array}{l}\text { Rapport des } 15 \text { ans } \\
\text { et moins sur la population } \\
\text { des } 65 \text { ans et plus. }\end{array}$ & $\mathrm{x}$ & \\
\hline \multirow{5}{*}{$\begin{array}{l}\text { Socio- } \\
\text { économique. . }\end{array}$} & $\%$ famille mono. & $\begin{array}{c}\text { Proportion des familles } \\
\text { monoparentales avec enfants } \\
\text { de moins de } 18 \text { ans. }\end{array}$ & $\mathrm{x}$ & \\
\hline & \% ménage 1 pers. & $\begin{array}{c}\text { Proportion des ménages } \\
\text { composés } \\
\text { d'une seule personne. }\end{array}$ & $\mathrm{x}$ & \\
\hline & \% bénéficiaire A.S. & $\begin{array}{l}\text { Proportion des bénéficiaires } \\
\text { de l'aide sociale. }\end{array}$ & $\mathrm{X}$ & \\
\hline & $\begin{array}{l}\text { \% pers. dans fam. } \\
\text { à faible revenu. }\end{array}$ & $\begin{array}{l}\text { Proportion des personnes } \\
\text { vivant dans des familles } \\
\text { à faible revenu. }\end{array}$ & $\mathrm{x}$ & \\
\hline & $\begin{array}{c}\text { Taux fécondité } \\
15-19 .\end{array}$ & $\begin{array}{l}\text { Taux de fécondité } \\
\text { des } 15-19 \text { ans. }\end{array}$ & $\mathrm{x}$ & \\
\hline \multirow{3}{*}{$\begin{array}{l}\text { Système soins et } \\
\text { services . . . . }\end{array}$} & Dép. encourues. & $\begin{array}{l}\text { Dépenses encourues } \\
\text { dans le programme. }\end{array}$ & & $\mathrm{X}$ \\
\hline & Hres travaillées. & $\begin{array}{l}\text { Heures travaillées } \\
\text { dans le programme. }\end{array}$ & & $\mathrm{X}$ \\
\hline & Densité pop. & Densité nette de la population. & & $\mathrm{X}$ \\
\hline \multirow{3}{*}{$\begin{array}{l}\text { Vitalité du } \\
\text { milieu }\end{array}$} & Nbre org. commu. & $\begin{array}{l}\text { Nombre d'organismes } \\
\text { communautaires per capita. }\end{array}$ & $\mathrm{X}$ & \\
\hline & Subv. org. commu. & $\begin{array}{l}\text { Subventions versées aux } \\
\text { organismes communautaires. }\end{array}$ & $\mathrm{X}$ & \\
\hline & Ratio (20-35) 86/81 & $\begin{array}{c}\text { Ratio des } 20-35 \text { ans en } 1986 \\
\text { sur le ratio } \\
\text { des } 20-35 \text { ans en } 1981 .\end{array}$ & & $\mathrm{X}$ \\
\hline \multirow{4}{*}{$\begin{array}{c}\text { Programme } \\
\text { personnes } \\
\text { âgées ... }\end{array}$} & $75+/ 65+$ & $\begin{array}{c}\text { Rapport des } 75 \text { ans et }+ \\
\text { sur le total des } 65 \text { ans et plus. }\end{array}$ & $\mathrm{x}$ & \\
\hline & $\% 65$ et + seules & $\begin{array}{c}\text { Proportion des personnes } \\
\text { de } 65 \text { ans et plus vivant seules. }\end{array}$ & $\mathrm{X}$ & \\
\hline & $\%$ Traumas $65+$ & $\begin{array}{l}\text { Proportion de traumatismes } \\
\text { chez les personnes âgées. }\end{array}$ & $\mathrm{x}$ & \\
\hline & $\begin{array}{l}\text { Taux préva. besoin } \\
\text { aide }\end{array}$ & $\begin{array}{l}\text { Taux de prévalence } \\
\text { des besoins d'aide. }\end{array}$ & $\mathrm{x}$ & \\
\hline
\end{tabular}

Note : Plusieurs de ces indicateurs étaient bien documentés par R. Choinière et al. [5]. Recherche opérationnelle/Operations Research 
ANNEXE 2

Modèle d'allocation personnes âgées en perte d'autonomie poids agrégés

\begin{tabular}{|c|c|c|c|c|c|}
\hline \multirow{2}{*}{ Dimension } & \multirow{2}{*}{ Poids } & \multirow{2}{*}{ Indicateur } & \multirow{2}{*}{ Poids } & \multicolumn{2}{|c|}{ Sens } \\
\hline & & & & Maximal & Minimal \\
\hline \multirow{3}{*}{ Démographique .... } & \multirow{3}{*}{.353} & Taux variation & .179 & $\mathrm{X}$ & \\
\hline & & Taille Pop. & .130 & & $\mathrm{X}$ \\
\hline & & Rapport $<15 />65$ & .044 & $\mathrm{X}$ & \\
\hline \multirow{5}{*}{ Socio-économique } & \multirow{5}{*}{.241} & $\%$ famille mono. & .075 & $\mathrm{X}$ & \\
\hline & & $\%$ ménage 1 pers. & .042 & $\mathrm{X}$ & \\
\hline & & \% bénéficiaire A.S. & .022 & $\mathrm{X}$ & \\
\hline & & $\begin{array}{l}\text { \% pers. dans fam. } \\
\text { à faible revenu. }\end{array}$ & .022 & $X$ & \\
\hline & & Taux fécondité 15-19. & .080 & $X$ & \\
\hline \multirow{3}{*}{ Système soins et services. } & \multirow{3}{*}{.168} & Dép. encourues. & .044 & & $X$ \\
\hline & & Hres travaillées. & .101 & & $\mathrm{X}$ \\
\hline & & Densité pop. & .023 & & $\mathrm{X}$ \\
\hline \multirow{3}{*}{ Vitalité du milieu . . . . } & \multirow{3}{*}{.106} & Nbre org. commu. & .078 & $\mathrm{X}$ & \\
\hline & & Subv. org. commu. & .020 & $\mathrm{X}$ & \\
\hline & & Ratio (20-35) 86/81 & .008 & & $X$ \\
\hline \multirow{4}{*}{$\begin{array}{l}\text { Programme personnes } \\
\text { âgées } \ldots \ldots \ldots\end{array}$} & \multirow{4}{*}{.131} & $75+/ 65+$ & .036 & $\mathrm{X}$ & \\
\hline & & $\% 65$ et + seules & .049 & $\mathrm{X}$ & \\
\hline & & $\%$ Traumas $65+$ & .010 & $\mathrm{X}$ & \\
\hline & & Taux préva. besoin aide & .036 & $\mathrm{X}$ & \\
\hline
\end{tabular}




\section{ANNEXE 3}

Enveloppe à répartir : $1012409 \$$

Allocation minimale : $55000 \$$

\begin{tabular}{|c|c|c|c|}
\hline M.R.C. & $\%$ & Rang & Allocation \\
\hline Kamouraska . . . . & 0,0 & 8 & $55000 \$$ \\
\hline La Mitis & 14,2 & 5 & $135952 \$$ \\
\hline Les Basques . . . . & 18,5 & 1 & $177121 \$$ \\
\hline Matane & 17,6 & 2 & $168504 \$$ \\
\hline Matapédia & 11,2 & 6 & $107230 \$$ \\
\hline R-du-Loup . . . . . & 6,7 & 7 & $64146 \$$ \\
\hline R-Neigette . . . & 16,8 & 3 & $160845 \$$ \\
\hline Temiscouata ... & 15,0 & 4 & $143611 \$$ \\
\hline
\end{tabular}




\begin{tabular}{|c|c|c|c|c|c|c|c|c|}
\hline & \multicolumn{8}{|c|}{ M.R.C. } \\
\hline $\begin{array}{l}\text { Indi- } \\
\text { cateurs }\end{array}$ & $\begin{array}{c}\text { Kamou- } \\
\text { raska }\end{array}$ & La Mitis & $\begin{array}{c}\text { Les } \\
\text { Basques }\end{array}$ & Matane & $\begin{array}{l}\text { Mata- } \\
\text { pédia }\end{array}$ & $\begin{array}{l}\text { R-du- } \\
\text { Loup }\end{array}$ & $\begin{array}{c}\mathrm{R}- \\
\text { Neigette }\end{array}$ & $\begin{array}{l}\text { Témis- } \\
\text { couata }\end{array}$ \\
\hline A & 0,07 & 0,35 & 1,00 & 0,27 & 0,12 & 0,14 & 0,48 & 0,40 \\
\hline B & $-1,9$ & $-1,8$ & $-1,5$ & $-2,2$ & $-2,3$ & $-2,2$ & $-2,6$ & $-2,4$ \\
\hline$C \ldots$ & 24520 & 21655 & 11155 & 25735 & 21575 & 30950 & 49095 & 25970 \\
\hline$D \ldots$ & 09,6 & 14,8 & 14,2 & 14,9 & 12,5 & 10,9 & 13,5 & 10,4 \\
\hline $\mathrm{E}$ & 16,5 & 17,5 & 17,1 & 17,0 & 14,2 & 17,3 & 18,0 & 15,3 \\
\hline $\mathrm{F}$ & 09,0 & 15,3 & 12,0 & 14,0 & 14,6 & 08,4 & 07,7 & 13,1 \\
\hline G $\ldots$ & 18,0 & 25,0 & 26,7 & 24,2 & 27,4 & 18,2 & 17,5 & 26,3 \\
\hline $\mathrm{H}$ & 2,66 & 4,02 & 2,33 & 4,81 & 5,23 & 4,19 & 2,20 & 3,24 \\
\hline I. & -4456 & -3500 & -3125 & -2437 & -2931 & -3567 & -2739 & -2522 \\
\hline $\mathrm{J}$ & $-110,0$ & $-095,8$ & $-071,3$ & $-061,8$ & $-073,3$ & $-087,6$ & $-068,3$ & $-062,8$ \\
\hline K & $-11,3$ & $-09,7$ & $-10,0$ & $-07,7$ & $-04,3$ & $-26,1$ & $-18,5$ & $-06,6$ \\
\hline$\cdots$ &, 05 &, 01 & ,08 &, 01 &, 02 &, 02 &, 02 &, 12 \\
\hline M & 0,08 & 1,72 & 1,76 & 0,51 & 1,91 & 0,24 & 0,55 & 0,28 \\
\hline $\mathrm{N}$ & $-098,43$ & $-100,00$ & $-095,63$ & $-098,58$ & $-107,75$ & $-096,75$ & $-095,44$ & $-107,35$ \\
\hline $\mathrm{O}$ &, 408 & ,398 & ,415 &, 372 &, 374 &, 373 &, 369 &, 353 \\
\hline$P$ & 13,3 & 18,0 & 16,7 & 18,6 & 18,0 & 14,5 & 17,6 & 14,3 \\
\hline Q & 05,1 & 06,0 & 07,0 & 04,3 & 06,3 & 05,1 & 04,7 & 05,5 \\
\hline$R$ & 741 & 648 & 371 & 690 & 587 & 849 & 1214 & 694 \\
\hline
\end{tabular}

Indicateurs : A, Taux variation; B, Taille Pop.; C, Rapport $<15 />65$; D, \% familles mono.; E, \% ménage 1 pers.; F, \% bénéficiaire A.S.; G, \% pers. dans fam. à faible revenu; H, Taux fécondité 15-19; I, Dép. encourues; J, Hres travaillées; K, Densité pop.; L, Nbre org. commu.; M, Subv. org. commu.; N, Ratio (20-35) 86/81; O, $75+/ 65+$; P, \% 65 et seules; Q, \% Traumas $65+; \mathrm{R}$, Taux préva. besoin aide. 